\title{
AIP
}

\section{Communication: Selection rules for tunneling splitting of vibrationally excited levels}

Willem Siebrand, Zorka Smedarchina, and Antonio Fernández-Ramos

Citation: J. Chem. Phys. 139, 021101 (2013); doi: 10.1063/1.4813002

View online: http://dx.doi.org/10.1063/1.4813002

View Table of Contents: http://jcp.aip.org/resource/1/JCPSA6/v139/i2

Published by the AIP Publishing LLC.

\section{Additional information on J. Chem. Phys.}

Journal Homepage: http://jcp.aip.org/

Journal Information: http://jcp.aip.org/about/about_the_journal

Top downloads: http://jcp.aip.org/features/most_downloaded

Information for Authors: http://jcp.aip.org/authors 


\title{
Communication: Selection rules for tunneling splitting of vibrationally excited levels
}

\author{
Willem Siebrand, ${ }^{1, a)}$ Zorka Smedarchina, ${ }^{1,2}$ and Antonio Fernández-Ramos ${ }^{2}$ \\ ${ }^{1}$ National Research Council of Canada, Ottawa, Ontario K1A OR6, Canada \\ ${ }^{2}$ Department of Physical Chemistry, Faculty of Chemistry and Centro Singular de Investigación \\ en Química Biolóxica e Materiales Moleculares (CIQUS), University of Santiago de Compostela, \\ 15706 Santiago de Compostela, Spain
}

(Received 31 May 2013; accepted 16 June 2013; published online 8 July 2013)

\begin{abstract}
Five symmetry-based selection rules are formulated that relate the tunneling splitting of a vibrationally excited level to that of the ground level in molecules with a symmetric double-minimum potential. The rules, which explain why excited levels frequently have smaller splittings than zero-point levels, are used to interpret the observed and calculated splittings in malonaldehyde.

(C) 2013 AIP Publishing LLC. [http://dx.doi.org/10.1063/1.4813002]
\end{abstract}

In this Communication we derive symmetry-based selection rules for tunneling splitting in vibrationally excited levels of polyatomic molecules and complexes, specifically rules that predict whether the splitting of an assigned level will be larger, smaller, or the same as that of the ground level, or, alternatively, which assignments are compatible with the observed splitting. For conciseness, we consider here only splitting due to two-fold symmetry corresponding to atomic tunneling in a symmetric double-minimum potential (SDMP). Our benchmark molecule will be malonaldehyde (MA), for which both experimental data ${ }^{1-3}$ and elaborate calculations ${ }^{4}$ are reported. These selection rules exist because tunneling is a quantum phenomenon; they invalidate the classical notion that putting energy into the well should make it easier to cross the barrier, which applies only when the energy is put directly into the tunneling vibration. As recent measurements of Suhm and co-workers ${ }^{3}$ and calculations of Hammer and Manthe ${ }^{4}$ on MA show abundantly, putting energy in other modes usually decreases the splitting, often dramatically, even for out-ofplane modes that have, at first sight, little to do with tunneling in this planar molecule. Here we show that this behavior can be understood on the basis of symmetry.

For a molecule with a SDMP, we use as reference configuration the transition state (TS), which is the point of highest symmetry, and divide the vibrations, obtainable from standard force field calculations, into symmetric and antisymmetric modes with respect to reflection in the dividing plane between the wells. The tunneling vibration is an antisymmetric mode and the other vibrations can only influence the tunneling to the extent that they are coupled to this mode. The extent of the coupling can be estimated from a comparison with the calculated harmonic force field of the equilibrium configuration (EQ) and TS; in the latter, the tunneling mode, taken as the reaction coordinate, is locally harmonic but with an imaginary frequency. Using a procedure previously introduced for instanton calculations, ${ }^{5}$ we expand the coupling in powers of the displacements of the coupled-mode coordinates of EQ relative to TS. If there are additional symmetry elements, as in

\footnotetext{
a)Electronic mail: willem.siebrand@nrc-cnrc.gc.ca
}

the case for MA, which is planar, there will be modes for which the linear coupling vanishes; this is the case for outof-plane modes in planar molecules such as MA. For the linearly coupled (i.e., displaced) modes, the effect of the coupling depends on the symmetry. For symmetric modes it gives rise to oscillation of the width and height of the barrier, while for antisymmetric modes, i.e., modes with the same symmetry as the tunneling mode, it generates a Franck-Condon factor. Thus the linear-coupling approximation, which keeps the overall force field harmonic, implies four symmetry-based selection rules that relate excited-level splittings to the groundlevel splitting:

Rule I: Excitation of the tunneling mode increases the tunneling splitting.

Rule II: Excitation of a symmetric mode increases the tunneling splitting.

Rule III: Excitation of an antisymmetric mode other than the tunneling mode decreases the tunneling splitting.

Rule IV: Excitation of an undisplaced mode has no effect on the tunneling splitting.

The extent of the increase or decrease is measured by the magnitude of the displacement and by the degree of excitation. However, for the present discussion we limit ourselves to fundamental excitations, since for overtones and combination bands other interactions may come into play.

Although some of these rules have been recognized occasionally, as, e.g., in our work on tropolone and 9hydroxyphenalenone, ${ }^{6}$ they are mostly ignored. That they present an incomplete picture is clearly shown by the splittings of excited levels of MA recently discussed in Refs. 1-4, as well as by the problems encountered by Havenith et al. ${ }^{7}$ in assigning splittings in the spectrum of the formic acid dimer (FAD). In the latter case, we showed ${ }^{8}$ that these problems can be traced back to the restriction to linear coupling. If quadratic coupling is included, all modes may couple to the tunneling mode and the overall force field will become anharmonic. Diagonal and off-diagonal coupling terms will then change frequencies and mix modes, and can be positive or negative. Since the strength of the overall force field 
will not change much between EQ and TS, there will be widespread cancellation between positive and negative terms in the ground state. By the same token, addition of nonlinear coupling terms to individual coupled modes is not expected to have a major effect of Rules I-IV.

However, the situation changes drastically if we consider bilinear coupling terms between different coupled modes, since such coupling may lead to exchange between the modes during the tunneling event. This was previously observed for the HF dimer. ${ }^{9}$ It also occurs in FAD, where its effect on the splitting could be calculated with good accuracy. ${ }^{8}$ This example proved to be of seminal value. The leading anharmonic term was found to be cubic and to involve coupling of a pair of undisplaced CO-stretch modes of different symmetry $\left(\mathrm{b}_{1 u}\right.$ and $\mathrm{b}_{2 u}$ in $\left.\mathrm{D}_{2 h}\right)$ with the tunneling mode $\left(\mathrm{b}_{3 g}\right)$, the tunneling being accompanied by interchange of these two modes. This interchange puts a drag on the transfer rate, thus reducing the splitting. The interchange implies a large cross term between the two modes. Characteristically, this term disappears, or nearly so, if we take sums and differences of the amplitude vectors, which transforms the two coupled normal modes into a pair of local modes ${ }^{10}$ that are identical except for being localized on different sides of the dividing plane. Exciting either of the normal modes therefore leads to a vibrational quantum ("exciton") that is quasilocalized on one side, its mobility being governed by the residual coupling between the two local modes. If this coupling is weak, it puts a damper on the simultaneous transfer of the tunneling particle and the vibrational exciton, and hence reduces the splitting. This, we emphasize, is not an incidental process special to FAD but a universal mechanism that occurs in all molecules or complexes in which tunneling splitting is governed by a SDMP. It imposes an additional selection rule:

Rule V: Excitation of any mode that interchanges with any other mode during the tunneling event, such that their combination is antisymmetric, decreases the tunneling splitting.

Although the apparent operation of such a rule has been observed previously, ${ }^{7-9}$ the symmetry-based origin of Rule $\mathrm{V}$ has not been recognized before. It is very powerful, not only for undisplaced modes, for which it will often overrule Rule IV, but also for symmetric and antisymmetric modes in cases were they show mixing, as illustrated in Fig. 1 for a pair of MA modes discussed below. It can therefore overrule even Rule II. Contrary to Rules I-IV, it is not limited to modes of a specified symmetry, but applies to all modes for which a suitable coupling partner is available.

To apply Rule V, we need to recognize pairs of modes that are strongly coupled. In lowest order it requires only knowledge of the structure and force field at TS, the most symmetric molecular configuration. From this information, we can find coupled modes $\left(i, i^{\prime}\right)$ by superimposing their amplitude vectors: Whenever this leads to sum and difference vectors that locate the vibration on one side of the dividing plane, a relevant local mode pair ${ }^{10}\left(j, j^{\prime}\right)$ has been identified. Such pairs of coupled normal modes, usually with similar frequencies, are ubiquitous in molecules with a SDMP because of the presence of a dividing plane. However, since it does not require mixing

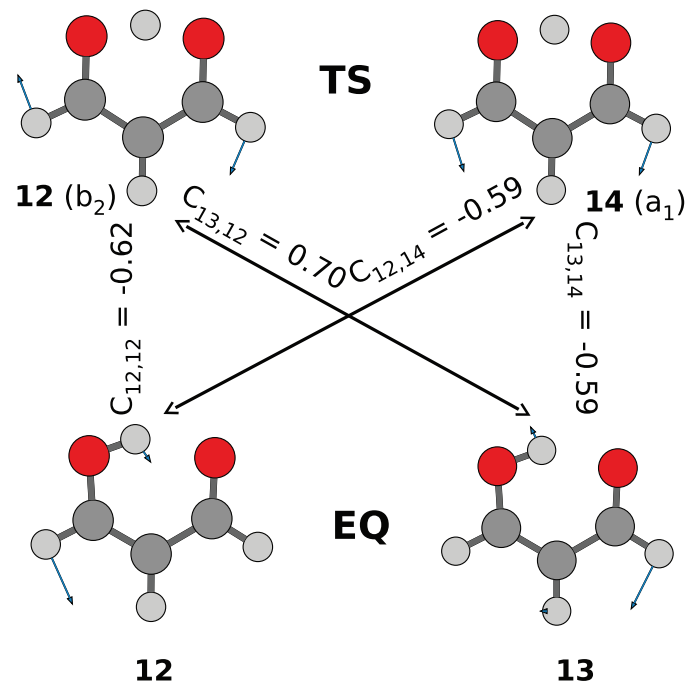

FIG. 1. Illustration of the anharmonic coupling that transforms normal modes $12\left(\mathrm{~b}_{2}\right)$ and $14\left(\mathrm{a}_{1}\right)$ in the transition state (TS) of malonaldehyde, to Modes 13 and 12 in the equilibrium configuration (EQ), which resemble local modes with amplitudes localized on either side of the dividing plane.

of equal parts, visual superposition may be occasionally misleading. A better strategy is to obtain quantitative information about the degree of mixing, and thus the relative strength of the suppression of the splitting, from the Dushinsky matrix between EQ and TS. If Mode $i$ in EQ is expanded in terms of the Modes $j$ in TS, a coupled pair $\left(i, i^{\prime}\right)$ will be characterized by

$$
\begin{aligned}
\left|c_{i, j}\right| & \simeq\left|c_{i^{\prime}, j}\right| \text { or }\left|c_{i^{\prime}, j^{\prime}}\right| ; \\
\left|c_{i, j^{\prime}}\right| & \simeq\left|c_{i^{\prime}, j^{\prime}}\right| \text { or }\left|c_{i^{\prime}, j}\right| ; \quad|i\rangle=\sum_{j} c_{i, j}|j\rangle,
\end{aligned}
$$

subject to the condition $\left|c_{i, j}\right|^{2}+\left|c_{i, j^{\prime}}\right|^{2} \sim 1$. The displacements of the symmetric and antisymmetric modes, as well as of the local modes, can be obtained from a comparison of the structures of EQ and TS, as previously shown for FAD. ${ }^{8}$

It follows that the present rules provide a framework for a general theory of excited level tunneling splittings. To complete the theory, a method is needed to turn the calculated displacements of the coupled normal and local modes into tunneling splittings. Earlier we have shown that the approximate instanton method (AIM) as implemented in the DOIT code ${ }^{11}$ is suitable for this purpose. A quantitative treatment of this type will be reported elsewhere.

To demonstrate the power of the selection rules, we apply them to MA, the usual benchmark for tunneling splittings, whose molecular symmetry group $\mathrm{G}_{4}$ corresponds to the point group $\mathrm{C}_{2 \mathrm{v}}$ of TS, the symmetry relevant for our purpose. The 21 normal modes belong to the representations $a_{1}$, $\mathrm{a}_{2}, \mathrm{~b}_{1}$, and $\mathrm{b}_{2}$. Note that $a_{2} \otimes b_{1}=b_{2}$, the symmetry of the tunneling mode. Following the work of Wilson et al. ${ }^{12}$ the vibrational force fields of both EQ and TS have been much studied, but some uncertainties remain. The conventional numbering of the normal modes in terms of increasing frequency may differ between authors and will differ also between EQ and TS. Here we use the harmonic force fields we recently adopted for our study of the zero-point splitting of MA. ${ }^{13}$ 
TABLE I. Comparison of Rules I-V with observed $\Delta_{i}^{\text {obs }}$ and calculated $\Delta_{j}^{\text {cal }}$ fundamental level splittings (in $\mathrm{cm}^{-1}$ ) in MA for all 21 Modes $i\left(i^{\prime}=\right.$ out-of-plane) in EQ. In TS modes are numbered by $j ; j$-values with superscripts + and - need to be changed by 1 to obtain the numbering of Ref. 4 , the source of $\Delta_{j}^{\text {cal }}$. The Dushinsky matrix coefficients are defined in Eq. (1). Unless otherwise indicated, $\Delta_{i}^{\text {obs }}$ values are taken from Ref. 3. The last column indicates the relevant normal-mode mixing when Rule $\mathrm{V}$ prevails.

\begin{tabular}{|c|c|c|c|c|c|c|c|c|}
\hline$i$ & $\omega_{i}$ & $j / \mathrm{sym}$ & $c_{i, j}$ & $c_{i, j^{\prime}}$ & $\Delta_{i}^{\mathrm{obs}}$ & $\Delta_{j}^{\mathrm{cal}}$ & Rule & $i / i^{\prime}$ \\
\hline $1^{\prime}$ & 255 & $2 / \mathrm{a}_{2}$ & $c_{1,2}=-0.91$ & $c_{1,3}=-0.40$ & $6-9$ & 6.7 & V & $1 / 3$ \\
\hline 2 & 271 & $5 / a_{1}$ & $c_{2,5}=-0.94$ & $c_{2,1}=-0.23$ & 57 & 64.0 & II & $\ldots$ \\
\hline $3^{\prime}$ & 399 & $3 / b_{1}$ & $c_{3,3}=0.91$ & $c_{3,2}=-0.39$ & 15 & 16.3 & V & $3 / 1$ \\
\hline 4 & 501 & $4 / b_{2}$ & $c_{4,4}=0.98$ & $c_{4,18}=0.15$ & 15 & 18.8 & III & $\ldots$ \\
\hline $5^{\prime}$ & 787 & $6 / b_{1}$ & $c_{5,6}=0.99$ & $c_{5,9}=0.12$ & $20-23$ & 21.1 & IV & $\ldots$ \\
\hline $6^{\prime}$ & 878 & $13^{-} / \mathrm{a}_{2}$ & $c_{6,13}=0.90$ & $c_{6,8}=0.36$ & $3-5$ & 2.7 & V & $6 / 9$ \\
\hline 7 & 885 & $7 / a_{1}$ & $c_{7,7}=0.95$ & $c_{7,5}=-0.24$ & 27 & 33.3 & II & $\ldots$ \\
\hline 8 & 982 & $10 / \mathrm{a}_{1}$ & $c_{8,10}=0.93$ & $c_{8,11}=-0.23$ & 14 & 14.6 & V & $8 / 11$ \\
\hline $9^{\prime}$ & 1017 & $8^{+} / b_{1}$ & $c_{9,8}=0.90$ & $c_{9,13}=-0.38$ & $\ldots$ & 7.5 & V & $9 / 6$ \\
\hline $10^{\prime}$ & 1043 & $9^{-} / \mathrm{a}_{2}$ & $c_{10,9}=0.96$ & $c_{10,8}=-0.23$ & $\ldots$ & $\ldots$ & IV & $\ldots$ \\
\hline 11 & 1100 & $11 / \mathrm{b}_{2}$ & $c_{11,11}=0.94$ & $c_{11,10}=-0.17$ & 17 & 19.5 & V & $11 / 8$ \\
\hline 12 & 1268 & $14 / \mathrm{a}_{1}$ & $c_{12,12}=-0.62$ & $c_{12,14}=-0.61$ & 8 & 2.9 & V & $12 / 13$ \\
\hline 13 & 1402 & $12^{+} / \mathrm{b}_{2}$ & $c_{13,12}=0.70$ & $c_{13,14}=-0.59$ & $\sim 0$ & $\ldots$ & V & $13 / 12$ \\
\hline 14 & 1407 & $18 / \mathrm{a}_{1}$ & $c_{14,18}=0.62$ & $c_{14,1}=0.48$ & $69 \pm 2$ & 83.6 & I & $\ldots$ \\
\hline 15 & 1463 & $15 / \mathrm{b}_{2}$ & $c_{15,15}=0.79$ & $c_{15,17}=0.37$ & $4-7$ & $\ldots$ & V & $15 / 17$ \\
\hline 16 & 1629 & $16^{+} / \mathrm{b}_{2}$ & $c_{16,16}=0.78$ & $c_{16,18}=-0.47$ & $21.55^{\mathrm{a}}$ & 6.5 & III & $\ldots$ \\
\hline 17 & 1702 & $17^{-/ \mathrm{a}_{1}}$ & $c_{17,17}=-0.85$ & $c_{17,15}=0.44$ & 7 & 18.8 & V & $17 / 15$ \\
\hline 18 & 2996 & $20 / a_{1}$ & $c_{18,20}=0.70$ & $c_{18,19}=0.70$ & $\ldots$ & $\ldots$ & V & $18 / 19$ \\
\hline 19 & 3181 & $19 / b_{2}$ & $c_{19,19}=0.70$ & $c_{19,20}=-0.68$ & 3 & $\ldots$ & V & $19 / 18$ \\
\hline 20 & 3223 & $21 / \mathrm{a}_{1}$ & $c_{20,21}=0.98$ & $c_{20,20}=0.14$ & 22 & $\ldots$ & II & $\ldots$ \\
\hline 21 & 3371 & $1 / b_{2}$ & $c_{21,1}=0.81$ & $c_{21,18}=-0.42$ & $\ldots$ & $\ldots$ & I & $\ldots$ \\
\hline
\end{tabular}

${ }^{a}$ Reference 2

Specifically, the geometries of the stationary points, as well as the Hessians are obtained at the MC-QCISD/3 level. ${ }^{14}$ The resulting EQ numbering $(i)$ and the corresponding symmetries and frequencies $\left(\omega_{i}\right.$, in $\left.\mathrm{cm}^{-1}\right)$ are listed in Table I along with the corresponding numbering $(j)$ of the TS modes; $j$-values with superscripts $+/-$ need to be changed by one unit to obtain the numbering of Ref. 4 . The Dushinsky matrix is represented by the two leading coefficients $c_{i, j}$ in Eq. (1) for each EQ mode; this is a good approximation only when the sum of the two squared coefficients is close to unity. The observed splittings $\Delta_{j}^{\text {obs }}$ are taken mostly from Ref. 3 and include new data that became available online only after the present work was completed. The calculated splittings $\Delta_{j}^{\text {cal }}$ are taken from Ref. 4 and refer to TS so that they are fully comparable to the observable splittings $\Delta_{i}^{\text {obs }}$ only when there is an exclusive 1:1 correspondence between the EQ and TS mode and when the calculations on the latter are fully converged. As indicated in Table XIII of Ref. 4, multiple correlations and partial convergence become a problem for levels with energies above $1000 \mathrm{~cm}^{-1}$. The column labeled Rule indicates which of Rules I-V governs the relative increase or decrease of the splitting compared to the observed zero-point splitting of $21.58 \mathrm{~cm}^{-1} .{ }^{15}$ The normal-mode couplings leading to local modes, listed in the last column, are derived from the Dushinsky matrix elements according to Eq. (1); observing splittings in the higher energy region is both more difficult and less informative than in the lower energy region.

Evidently, Rules I-V all find application and together they account for all the observed relative splittings. The data for Modes $i=1-3,7,8,11,17,19$, and 20 amount to con- firmed predictions, since they became available only after submission. ${ }^{3}$ The comparison with the calculated splittings of Hammer and Manthe ${ }^{4}$ is also satisfactory for levels below about $1400 \mathrm{~cm}^{-1}$. Evidently, accurate results can be obtained with direct diagonalization when the calculations are converged, but the computational effort required seems to limit the applicability of the method to relatively low energy levels and small systems, as suggested by the results for Modes $j=16$ and 17 , reported as not fully converged. ${ }^{4}$

Modes $i=2,7$, and 14 are $\mathrm{a}_{1}$ modes with a large, modest, and again modest displacement, respectively, as reflected in their calculated splittings; however, on the basis of the Dushinsky matrix, we assign the large splitting of Mode 14 predominantly to direct linear coupling to the tunneling mode. The smallness of the splitting of the undisplaced fundamentals $i=1,3,6$, and 9, due to pairwise coupling of (undisplaced) out-of-plane modes, stands out. On the other hand, the out-of-plane Mode $i=5$, which is not coupled to a complementary partner, has the same splitting as the zero-point level, as predicted by Rule IV. Pairwise coupling between a symmetric and an antisymmetric in-plane mode is seen for Modes $i=12$ and 13, illustrated in Fig. 1, as well as for Modes 8 and 11, 15 and 17, and 18 and 19. In these cases Rule V overrules Rule II. In our calculation, Mode $i=16$ is not significantly displaced and not partnered with another vibration to form a local mode; hence its splitting should be similar to that of the ground level, in agreement with experiment but contrary to the result in Ref. 4. Mode $i=20$ is a symmetric $\mathrm{CH}$-stretching mode that is not displaced and not significantly mixed with any other mode; hence it should have 
basically the same splitting as the ground level, as indeed observed.

Table I shows that of the 21 fundamentals of MA nine are governed by the linear-coupling Rules I-IV and 12 by the bilinear-coupling Rule V. It follows that Rule V dominates the excited level splittings in MA, which is the reason why most of these splittings are smaller than the zero-point splitting. We finally point out that these symmetry-based selection rules provide an additional tool for assigning spectra exhibiting tunneling splitting, an early example being the reassignment of the observed FAD splitting. ., $^{\circ}$

We are grateful to an anonymous reviewer for his helpful comments and for drawing our attention to a very recent online publication with relevant new data. This work was partially financed by the Xunta de Galicia through Grant No. 2012/314.

${ }^{1}$ C. J. Seliskar and R. E. Hoffmann, J. Mol. Spectrosc. 96, 146 (1982). ${ }^{2}$ C. Duan and D. Luckhaus, Chem. Phys. Lett. 391, 129 (2004).

${ }^{3}$ T. N. Wassermann, D. Luckhaus, S. Coissan, and M. A. Suhm, Phys. Chem. Chem. Phys. 8, 2344 (2006); N. O. B. Lüttschwager, T. N. Wassermann, S. Coissan, and M. A. Suhm, ibid. 12, 8201 (2010); "Vibrational tuning of the hydrogen transfer in malonaldehyde - a combined FTIR and Raman study," Mol. Phys. (to be published).

${ }^{4}$ T. Hammer and U. Manthe, J. Chem. Phys. 136, 054105 (2012).

${ }^{5}$ Z. Smedarchina, W. Siebrand, M. Z. Zgierski, and F. Zerbetto, J. Chem. Phys. 102, 7024 (1995); Z. Smedarchina, W. Siebrand, and M. Z. Zgierski, ibid. 103, 5326 (1995).

${ }^{6}$ Z. Smedarchina, W. Siebrand, and M. Z. Zgierski, J. Chem. Phys. 104, 1203 (1996); A. Fernández-Ramos, Z. Smedarchina, M. Z. Zgierski, and W. Siebrand, ibid. 109, 1004 (1998).

${ }^{7}$ F. Madeja and M. Havenith, J. Chem. Phys. 117, 7162 (2002); M. Ortlieb and M. Havenith, J. Phys. Chem. A 111, 7355 (2007).

${ }^{8}$ Z. Smedarchina, W. Siebrand, and A. Fernández-Ramos, Chem. Phys. Lett. 395, 339 (2004).

${ }^{9}$ A. S. Pine, W. J. Lafferty, and B. J. Howard, J. Chem. Phys. 81, 2939 (1984).

${ }^{10}$ B. R. Henry and W. Siebrand, J. Chem. Phys. 49, 5369 (1968).

${ }^{11}$ W. Siebrand, Z. Smedarchina, M. Z. Zgierski, and A. Fernández-Ramos, Int. Rev. Phys. Chem. 18, 5 (1999); Z. Smedarchina, A. Fernández-Ramos, and W. Siebrand, J. Comput. Chem. 22, 787 (2001).

${ }^{12}$ S. L. Baughcum, Z. Smith, E. B. Wilson, and R. W. Duerst, J. Am. Chem. Soc. 106, 2260 (1984).

${ }^{13}$ Z. Smedarchina, W. Siebrand, and A. Fernández-Ramos, J. Chem. Phys. 137, 224105 (2012).

${ }^{14}$ B. J. Lynch and D. G. Truhlar, J. Phys. Chem. A 107, 3898 (2003).

${ }^{15}$ D. W. Firth, K. Beyer, M. A. Dvorak, S. W. Reeve, A. Q. Gushov, and K. Leopold, J. Chem. Phys. 94, 1812 (1991); T. Baba, T. Tanaka, I. Morino, K. M. T. Yamada, and K. Tanaka, ibid. 110, 4131 (1999). 